\title{
Brain responses in CFS and TMD to autonomic challenges: An exploratory fMRI study
}

Quoc C. Vuong: Institute of Neuroscience, Newcastle University, Framlington Place, Newcastle upon Tyne, NE2 4HH, UK; email: quoc.vuong@newcastle.ac.uk; phone: +44 (0)191 2086183

James R. Allison: School of Dental Sciences, Newcastle University, Framlington Place, Newcastle upon Tyne, NE2 4BW, UK; email: james.allison@newcastle.ac.uk

Andreas Finkelmeyer: Institute of Neuroscience, Newcastle University, Framlington Place, Newcastle upon Tyne, NE2 4HH, UK; email: andreas.finkelmeyer@newcastle.ac.uk

Julia Newton: Institute of Cellular Medicine and NIHR Newcastle Biomedical Research Centre, Newcastle University, Newcastle upon Tyne, NE2 4HH, UK; Newcastle Hospitals NHS Foundation Trust, Newcastle upon Tyne, NE7 7DN, UK; email: julia.newton@newcastle.ac.uk

Justin Durham: School of Dental Sciences, Newcastle University, Framlington Place, Newcastle upon Tyne, NE2 4BW, UK; Institute of Health and Society, Newcastle University, Newcastle upon Tyne, NE2 4AX, UK; Dental Hospital, Newcastle Hospitals NHS Foundation Trust, Newcastle upon Tyne, NE2 4AZ, UK email: justin.durham@newcastle.ac.uk

Abstract word count: 262

Manuscript word count: 3989

Total number of figures/tables: 3 figures/2 tables

Total number of references: 39

Keywords: Chronic Fatigue Syndrome; Temporomandibular Disorders; Autonomic Nervous System Diseases; Dysautonomia; Magnetic Resonance Imaging; Valsalva Manoeuvre 


\section{Knowledge Transfer Statement}

Brain activity related to activation of the autonomic nervous system in chronic fatigue syndrome patients who screened positive for painful temporomandibular disorder was greater than in patients who screened negatively; activity was seen in brain regions associated with autonomic functions and pain. These findings suggest that autonomic dysfunction may play a role in the pathophysiology of both conditions, explain some of the apparent co-morbidity between the two, and offer avenues to help with treatment.

\section{Abstract}

INTRODUCTION: Dysfunction of the autonomic nervous system (ANS) is seen in chronic fatigue syndrome (CFS) and temporomandibular disorders (TMD). Both conditions have poorly understood pathophysiology. Several brain structures which play a role in pain and fatigue, such as the insular cortex and basal ganglia, are also implicated in autonomic function.

OBJECTIVES: ANS dysfunction may point to common neuro-physiological mechanisms underlying the predominant symptoms for both CFS and TMD. No studies to date have investigated the combination of both conditions. Thus our aim was to test whether CFS patients with or without TMD show differences in brain responses to autonomic challenges.

METHODS: In this exploratory functional imaging study, CFS patients who screened positive for $\operatorname{TMD}(n=26)$, patients who screened negative for TMD $(n=16)$ and age-matched control participants $(n=10)$ performed the Valsalva manoeuvre whilst in a 3T MRI scanner. This manoeuvre is known to activate the ANS.

RESULTS: For all three groups, whole-brain F-test showed increased brain activation during the manoeuvre in superior and inferior frontal gyri, left and right putamen and thalamus, and insular cortex. Furthermore, group contrasts with small-volume correction showed that CFS patients who screened positive for TMD showed greater activity in the left insular cortex compared to patients who screened negative, and in the left caudate nucleus compared to controls.

CONCLUSION: Our results suggest that increased activity in cortical and subcortical regions observed during autonomic challenges may be modulated by fatigue and pain. ANS dysfunction may be a contributing factor to these findings and further work is required to tease apart the complex relationship between CFS, TMD and autonomic functions. 


\section{Introduction}

The pathophysiology of chronic fatigue syndrome (CFS) remains poorly understood. In practice, it is clinically characterised by debilitating fatigue lasting at least six months, alongside a host of cooccurring symptoms such as poor sleep, post-exertional malaise, muscle and joint pain, and reduced cognitive capacity. The gold standard diagnostic criteria allow for any combination of at least four out of eight symptoms (Fukuda et al. 1994); consequently, there are many possible permutations. The resulting symptom clusters alongside the predominant symptom of pain and fatigue may reflect different CFS phenotypes, for example, due to other co-morbid conditions. The different phenotypes may have different underlying pathogenesis, which may increase the difficulty of finding appropriate treatments.

Despite the potential heterogeneity of CFS, nearly $90 \%$ of patients with CFS have associated autonomic nervous system (ANS) dysfunction (Newton et al. 2007; Robinson et al. 2015; Van Cauwenbergh et al. 2014). The ANS may contribute to common neuro-physiological mechanisms underlying some of the diagnostic symptoms of CFS, particularly pain and fatigue (Newton et al. 2007). Thus the aim of our exploratory study is to test whether there are differences in brain responses to autonomic challenges between two possible CFS phenotypes - patients who also screen positive for painful Temporomandibular Disorders (TMD) and patients who screen negative for TMD. Other clinical pain conditions in the absence of CFS, such as migraines or fibromyalgia, may also be associated with ANS dysfunction (Borsook et al. 2016; Martinez-Martinez et al. 2014).

Based on our previous studies (Finkelmeyer et al. 2018a; Finkelmeyer et al. 2018b; Robinson et al. 2015), we focused on TMD in the current brain imaging study for several reasons. First, differences in TMD patients' heart rate variability compared to controls during rest and orthostatic challenge have been observed, which correlated with the degree of pain sensitivity (Chinthakanan et al. 2018; Maixner et al. 2011). Heart rate variability can be an indicator of autonomic dysfunction (Robinson et al. 2015). Second, increased resting heart rate variability increased the likelihood of TMD in a large prospective cohort study (Greenspan et al. 2013). Third, there is a higher prevalence of TMD in patients with CFS, and vice versa, than the reported population prevalence (Johnston et al. 2013; Robinson et al. 2016; Slade et al. 2013). Lastly, ANS dysfunction is greater in CFS patients who screened positive for TMD compared to those who screened negative for TMD (Robinson et al. 2015). It is possible therefore that the presence of TMD in CFS indicates a potential phenotype with a greater burden on the ANS.

Centrally both cortical and subcortical structures are involved in autonomic functions, including the insular cortex (Macey et al. 2012; Seifert et al. 2013) and basal ganglia (Miller et al. 2014; Pazo and Belforte 2002). Structures within the basal ganglia also have a role in fatigue (Miller et al. 2014), and the insular cortex is part of a network of cortical regions involved in pain processing (Brugger et al. 2012; Harte et al. 2016; Seifert et al. 2013). The insular cortex is particularly interesting because imaging studies with CFS and TMD patients show anatomical and functional differences between each patient group and control groups in this region (Finkelmeyer et al. 2018b; 
Miller et al. 2014; Younger et al. 2010). Thus given their potential role in both autonomic functions and pain processing, we hypothesise that brain responses to autonomic challenges in the insular cortex and basal ganglia may be modulated by the presence of CFS and TMD.

Building on our previous work with the same cohort of patients (Finkelmeyer et al. 2018a; Finkelmeyer et al. 2018b; Robinson et al. 2015), we used functional magnetic resonance imaging (fMRI) to measure whether different CFS phenotypes (i.e., presence or absence of TMD) show functional brain differences related to autonomic functions. This is an important aim because not all studies find a link between CFS and ANS dysfunctions (Robinson et al. 2015; Timmers et al. 2002). Similarly, there are discrepancies in whether researchers find cerebral anatomical differences between individuals with and without CFS (Finkelmeyer et al. 2018b; Puri et al. 2012; van der Schaaf et al. 2017). The different findings reported in previous studies may be a consequence of diverse CFS symptom clusters or failure to control for other co-morbidities. Thus in the current study, we compared brain activation in response to an autonomic respiratory challenge (Valsalva manoeuvre) between CFS patients who screened positive or negative for TMD. This manoeuvre is used clinically to induce changes in systemic blood pressure and heart rate. Given our hypothesis, we expect blood oxygenation-level dependent (BOLD) responses to differ between the two CFS groups during this manoeuvre, and between the patient groups and the control group. We further expect to observe these differences in insular cortex (Brugger et al. 2012; Finkelmeyer et al. 2018a; Harte et al. 2016), although other brain regions that are involved in autonomic function or pain processing may also show differences (Seifert et al. 2013).

\section{Methods}

\section{Participants}

A total of 52 participants were tested in the current study, which included the same cohort groups from our previous studies (Finkelmeyer et al. 2018a; Finkelmeyer et al. 2018b; Robinson et al. 2015). Table 1 provides a breakdown of participant information per group. CFS patients were recruited via the Newcastle and North Tyneside National Health Service Clinical CFS Service as part of a larger project investigating the role of autonomic dysfunctions in CFS. They were included if they fulfilled the Fukuda diagnostic criteria for CFS (Fukuda et al. 1994) and screened negative for current or past major depressive episodes, as assessed by a trained medic using the Structured Clinical Interview for the Diagnostic and Statistical Manual for Mental Disorders (version IV; SCID-IV (First et al. 2002)). We used a TMD screening instrument (Gonzalez et al. 2011) to screen for TMD. This instrument is a 6-item self-report questionnaire and has very high sensitivity and specificity for painful TMD (>95\%). Patients filled out the DePaul Symptom Questionnaire (DSQ; (Jason et al. 2010)), the Fatigue Impact Scale (FIS; (Fisk et al. 1994)), and the Pain Catastrophizing Questionnaire (PCQ; (Sullivan et al. 1995)). Age-matched participants for the control group were recruited as part of the same larger project; they had no diagnoses of CFS, screened negative for TMD, and no history of major psychiatric and physical health conditions. Participants underwent a medical assessment at 
the time of recruitment that identified that they did not have other diagnoses. Thus there were three groups: CFS patients who screen positive for TMD (screener score $\geq 3$ ), CFS patients who screen negative for TMD (screener score $\leq 2$ ), and age-matched participants without CFS diagnoses and screen negative for TMD (screener score=0). These will be referred to as the CFS+, CFS- and Control group, respectively. All participants provided written informed consent. The study was conducted in accordance with the Declaration of Helsinki. The study was reviewed and approved by the local NHS Research Ethics Committee (REC 12/NE/0146, CLRN ID 97805).

\section{--- Insert Table 1 about here ---}

\section{Image acquisition during the Valsalva manoeuvre}

A T1-weighted anatomical scan was collected for each participant using a 3D MPRAGE sequence $\left(\mathrm{TE}=4.6 \mathrm{~ms} ; \mathrm{TR}=8.3 \mathrm{~ms}\right.$; flip angle $=8^{\circ}$; three-dimensional acquisition field of view $(\mathrm{FOV})=240$ $\mathrm{mm}(\mathrm{AP}) \times 216 \mathrm{~mm}(\mathrm{FH}) \times 180 \mathrm{~mm}(\mathrm{LR}) ; 1 \mathrm{~mm}$ isotropic voxel size). These scans were processed and analysed in our previous study (Finkelmeyer et al. 2018b), so are not reported further.

In the same scanning session, functional $\left(\mathrm{T}^{*}\right)$ echo planar images (EPI) were acquired in a single functional run while participants performed four Valsalva manoeuvres. Figure 1 (panel A) illustrates the time course of the functional scan and the periods of interest for our analyses. The time course consisted of a 4-s preparation period (light grey region), a 16-s Valsalva period and a 40-s rest period, repeated four times. The rest period was divided into a 20 -sec post-Valsalva period for the purpose of analyses (dark grey region; see Figure 1, panel A). We followed our previous protocol for MRI acquisition during the Valsalva manoeuvre (Bohr et al. 2015). Participants were guided through the protocol using a projection screen. First, they were presented with visual instructions to inhale before the manoeuvre during the preparation period, and then to start and stop exhaling during the Valsalva period. During the Valsalva period, participants maintained an expiratory pressure of $40 \mathrm{mmHg}$ against a closed outlet which provided a resistance so that their expiratory pressure could be measured using an in-house device and software. Participants received visual feedback (a bar chart) of the measured expiratory pressure to help them maintain this target pressure. All participants in this study were able to maintain the target pressure. Figure 1 (panel B) illustrates CFS+ patient's exhaled pressure trace. Participants were scanned in a 3 Tesla Philips Intera Achieva scanner using an eight-channel head coil. We used a customised dual gradient echo sequence to acquire 135 images (repetition time [TR] = $2000 \mathrm{~ms}, 270 \mathrm{sec}$ total data acquisition; echo times [TE1/TE2] = 13.82/39.27 ms; flip angle $=90^{\circ}$; two-dimensional acquisition FOV $=240$ $\mathrm{mm} \times 240 \mathrm{~mm}$; matrix size = 112 pixels $\times 112$ pixels; and 20 slices of 4 -mm thickness).

--- Insert Figure 1 about here --- 


\section{fMRI pre-processing}

The functional images (135 images) were realigned to the mean of all functional images and directly normalised to a standard Montreal Neurological Institute (MNI) T2*-weighted template with a resampled voxel size of $3 \mathrm{~mm} \times 3 \mathrm{~mm} \times 3 \mathrm{~mm}$. The normalised images were spatially smoothed with a $6 \mathrm{~mm}$ at full-width-half-maximum Gaussian kernel to improve the signal-to-noise ratio and to allow for comparisons across participants. We then applied a high-pass filter with a cut-off of $128 \mathrm{~s}$ to remove low-frequency drifts in the signal, and used an autoregressive model to estimate serial correlations in the data and adjust degrees of freedom accordingly.

\section{fMRI whole brain analyses}

The pre-processed data were analysed using SPM12 (Friston et al. 1994). We used the general linear model (GLM) with a two-step mixed-effects approach. First, a fixed-effects model was used to analyse each participant's data set. Second, a random-effects model was used to analyse the individual datasets at the group level. No additional smoothing of the images was used at the group level.

At the subject level, we first created a design matrix for each participant as follows. The preparation, Valsalva and post-Valsalva periods were each modelled as box-car time series and convolved with a canonical hemodynamic response function. The remaining time points were unmodelled and served as an implicit baseline. The global signal (i.e., the mean time series computed over all grey-matter voxels and the six movement parameters from the realignment were included as regressors of no interest in the matrix. We then fitted a linear combination of the regressors to the functional data to estimate beta weights for each period and create a corresponding beta image. Lastly, we calculated a contrast image between the Valsalva and the other two periods from their corresponding beta images (i.e., Valsalva - [preparation + post-Valsalva]). The resulting value at each voxel in the contrast image reflects the magnitude of brain activation during the Valsalva manoeuvre at that voxel.

At the group level, we conducted a series of analyses to compare contrast images between the CFS+, CFS- and Control groups. First, we conducted an F-test to localize clusters in which any of the three groups showed beta weights significantly greater than zero. For this test, we used an initial (uncorrected) threshold of $p<0.0001$ and a minimum cluster size of $k=100$ voxels. Second, we conducted whole-brain pairwise $t$-tests between groups using an initial (uncorrected) threshold of $p<0.01$ and a minimum cluster size of $k=10$ voxels. Based on our hypotheses that insular cortex and basal ganglia may be involved in autonomic functions, pain and fatigue, we used significant clusters from the F-test which overlapped with these anatomical regions as separate masks for posthoc small volume correction (SVC) to increase statistical power for our group comparisons. Lastly, we report clusters which had $p$ (uncorrected) $<0.05$ at the cluster level. We do not consider these uncorrected clusters as significant and only present them for exploratory purposes. 


\section{Results}

Brain regions showing consistent increased activation across groups

Figure 2 and Table 2 show the significant clusters from the $F$-test. There is increased brain activation in several cortical and subcortical regions when participants performed the Valsalva manoeuvre (Figure 2, panel A). The cortical clusters included those in superior and inferior frontal gyri. The subcortical clusters were centred within left and right putamen and thalamus. Part of the putamen clusters overlapped with insular cortex (Figure 2, panel B; left hemisphere: 59 voxels, 16.0\%; right hemisphere: 23 voxels, 7.5\%; based on the WFU PickAtlas; http://fmri.wfubmc.edu/software/PickAtlas, version 2.4; (Maldjian et al. 2003)).

--- Insert Figure 2 about here ---

--- Insert Table 2 about here ---

Brain regions showing differences between CFS patients who screened positive or negative for TMD Figure 3 and Table 2 show the clusters from whole-brain pairwise $t$-tests between the CFS+ and CFS-group. These comparisons tested whether the presence of TMD in CFS patients affected brain activity during the Valsalva manoeuvre. Only the left cluster from the $F$-test centred on the putamen overlapped with the uncorrected clusters from this contrast. After small volume correction with this cluster, there was one cluster within left insular cortex which showed significantly larger BOLD responses for the CFS+ group compared to the CFS- group. To facilitate future studies, we computed the effect size of this group difference as follows. For each participant in each group we first calculated the mean beta value for the cluster (i.e., we averaged across the 26 voxels in the cluster from the contrast image: Valsalva - [preparation + post-Valsalva]), and then we calculated Hedges' $g$ (for unequal group sample sizes) as our measure of effect size. For the CFS+ group, the mean beta value averaged across participants was 0.70 (arbitrary units), with a standard deviation of 0.51 . For the CFS- group, the mean beta value was 0.13 , with a standard deviation of 0.41 . We found a large effect size (Hedges' $g=1.27 ; 95 \%$ confidence interval: CFS+ [0.43 0.97], CFS- [-0.03 0.30]). We did not find any significant clusters for the opposite contrast: there were no clusters showing larger responses for the CFS- compared to the CFS+ group.

--- Insert Figure 3 about here ---

Brain regions showing differences between CFS patients and age-matched participants Next we compared each patient group to the Control group using whole-brain pairwise $t$-tests. For the comparison between the CFS+ and Control groups, there was one cluster within left caudate showing significantly larger BOLD responses for patients than controls (after small volume correction). The caudate is part of the basal ganglia, which is involved in autonomic functions (Miller 
et al. 2014; Pazo and Belforte 2002) and may play a role in fatigue (Miller et al. 2014). For the CFS+ group, the mean beta value averaged across participants was 0.94 , with a standard deviation of 0.65. For the Control group, the mean beta value was -0.33 , with a standard deviation of 0.84 . We found a large effect size also for this cluster (Hedges' $g=1.75 ; 95 \%$ confidence interval: CFS+ [0.60 1.29], Control [-0.93 0.27]). There were no significant clusters within insular cortex for the comparison between the CFS- and Control group, although there were clusters in other cortical regions for this comparison ( $p<0.05$, uncorrected at the cluster level). These include clusters in the cingulate cortex, which is a region that also plays a role in pain processing (Boccard et al. 2014; Schmidt-Wilcke et al. 2014). Finally, there were no significant clusters in which BOLD responses were significantly larger for the Control group compared to either patient groups.

\section{Discussion}

In the current fMRI study, brain activity was measured whilst patients and healthy participants performed the Valsalva manoeuvre to cause autonomic activation. Brain activity was compared between three groups: CFS patients who screened positive for TMD; those who screened negative for TMD; and age-matched participants who did not have a CFS diagnosis and screened negative for TMD (i.e., a control group). None of the participants had any current or previous major depressive episodes, or other major medical conditions. Consistent with previous work (Henderson et al. 2002; Macey et al. 2012), there was increased brain activity during the Valsalva manoeuvre across all three groups within left and right putamen with voxels overlapping insular cortex (Figure 2, panel B; Table 2). There was also increased brain activity in the thalamus and frontal lobe. In addition, CFS patients who screened positive for TMD showed: (1) increased brain activity during the Valsalva manoeuvre in a cluster within left insular cortex relative to CFS patients who screened negative for TMD; and (2) increased brain activity in left caudate relative to healthy controls (Table 2). By comparison, there were some preliminary evidence that CFS patients who screened negative for TMD showed increased brain activity only in temporal and frontal cortices during the Valsalva manoeuvre relative to controls. The functional differences between groups reported here complement differences in grey matter volume previously reported for the same patient cohorts (Finkelmeyer et al. 2018b). In that study, CFS patients showed increased grey matter volume in insula, temporal and frontal gyri, and thalamus relative to healthy controls.

The insular cortex has a known role in pain processing (Harte et al. 2016; Seifert et al. 2013), and-together with the limbic system-form part of the higher brain functioning pathway for ascending orofacial nociception beginning in the Trigeminal peripheral first order afferents. The results from the current study suggest that the increase in insula activity in response to the Valsalva manoeuvre may be amplified by the additional orofacial pain in CFS patients who screened positive for TMD compared to those who screened negative for TMD. That is, the Valsalva manoeuvre engages the autonomic system resulting in insula activity (across all groups). This activity is amplified in CFS+ patients by the additional orofacial pain they experience. The difference between the two 
patient groups was found only in the left insula. This hemispheric asymmetry may be related to the finding that insula responses to the Valsalva manoeuvre in healthy participants is larger in the left compared to the right hemisphere (Macey et al. 2012), which may allow for more differential changes between the two patient groups to be detected.

Previous functional imaging studies have shown increased brain activity in insular cortex during the Valsalva manoeuvre in healthy cohorts, consistent with this region's role in autonomic functions (Henderson et al. 2002; Macey et al. 2012). Although we hypothesised that ANS dysfunction may contribute to CFS (Newton et al. 2007; Robinson et al. 2015; Van Cauwenbergh et al. 2014), we only found a significant difference in left caudate between CFS patients who screened positive for TMD and control participants. The caudate is part of the basal ganglia, which may be involved in autonomic functions and fatigue (Miller et al. 2014; Pazo and Belforte 2002). The combination of CFS and TMD may increase the burden on the ANS for these patients, leading to the observed functional difference in the caudate nucleus. We did not find significant differences in brain activity in insula between either of the CFS patient group and the control group. We do not know why this was the case, although the combination of the small number of controls and unequal sex distribution across the three groups may be contributing factors. There were some preliminary functional differences in other cortical structures between CFS patients who screened negative for TMD and control participants (Table 2).

The Valsalva manoeuvre elicits both sympathetic and parasympathetic responses (Macey et al. 2012). The findings from the current study cannot pinpoint which system (or both) may be driving the functional differences observed between our participant groups. That said, an earlier study with the same patient cohort found that CFS patients with TMD (screened positive) showed evidence of greater dominance of the parasympathetic nervous system relative to control (Robinson et al. 2015). By comparison, CFS patients without TMD (screened negative) showed no differences to control on heart rate variability measures related to both of these systems. These findings reflect dysfunction of the sympathetic nervous system, with increased parasympathetic activity compensating for chronic elevated levels of sympathetic activity (Robinson et al. 2015). Related to this, studies of heart rate variability in patients with only TMD found similar elevated level of sympathetic activity (Chinthakanan et al. 2018; Maixner et al. 2011). Future studies with CFS patients could use other autonomic challenges such as the hand grip challenge, which is a non-painful sympathetic challenge, to further refine our understanding of the role of the insular cortex in CFS. It is worth noting that the Valsalva manoeuvre activates body receptors in the somatosensory pathway which may be affected by pain conditions; thus the results reported may relate to differences in somatosensory sensitivity rather than autonomic activity per se (Khurana 2014). Future studies are required to tease apart autonomic and somatosensory activity (e.g., using somatosensory amplification scale as a co-variate measure (Koteles and Witthoft 2017)).

Taking into consideration the results across the studies with the same participant cohort (Finkelmeyer et al. 2018a; Finkelmeyer et al. 2018b; Robinson et al. 2015), we have identified 
several measures from heart rate variability to anatomical and functional measurements that discriminate between potential CFS phenotypes; in our case, CFS patients who screen positive or negative for TMD. These measures in combination point to the importance of autonomic functions for moderating fatigue and pain-two diagnostic symptoms of CFS. Thus, treatments which modulate these functions may help patients manage their symptoms more effectively. For example, beta-blockers may be an effective pharmacological intervention (Light et al. 2009). Psychosocial interventions may also be effective; for example, diaphragmatic breathing exercises may help harness the innate, inbuilt functionality of the Vagus nerve to increase parasympathetic activity to help CFS patients with and without co-morbid TMD cope with persistent pain (Bordoni et al. 2016). If there is no intervention, a negative feedback loop may develop as more persistent pain will increase negative affect and reinforce ANS dysfunction which will subsequently worsen pain experience.

There are a number of limitations in the current study. First, we must bear in mind differences in sample size across the three groups in the current study, particularly the small number of controls. There was also a different ratio of females to males in each group. Second, we did not know the participants' pain state while they were being scanned (or any painful episodes they may have experienced on the day of the scan). However, CFS patients who screened positive for TMD have higher self-reported pain experiences (as measured by the pain-related questions from the DSQ scores) compared to patients who screened negative for TMD (Table 1). That said, these limitations may contribute to the non-significant functional differences between groups. Third, our patient recruitment and screening excluded those with other medical conditions that share symptoms with CFS, such as migraines and fibromyalgia, which may also be the result of ANS dysfunction (Borsook et al. 2016; Light et al. 2009; Martinez-Martinez et al. 2014; Schmidt-Wilcke et al. 2014). Similar to CFS, fibromyalgia, in particular, affects predominantly females and leads to fatigue and pain symptoms. Lastly, we did not confirm our TMD screening with clinical examination so it is likely that there was a heterogeneous sample in terms of painful TMD; we therefore do not know whether the orofacial pain was of a muscular or arthrogenous origin, and whether it was acute or chronic in onset and duration.

\section{Conclusion}

In the current study, functional differences in cortical and subcortical regions between CFS patient groups and between patient and control groups were found during ANS activation. Although there were limitations in our exploratory study, it is the first study to scan a relatively large number of CFS patients, while controlling for additional pain conditions and depression. Given our promising results, future work can use similar fMRI paradigms to systematically compare and contrast brain responses to parasympathetic and sympathetic autonomic challenges across different clinically-diagnosed patient groups that share pain and fatigue as major symptoms. 


\section{Acknowledgments}

This study and JN were funded by an MRC research grant (MR/J002712/1).

\section{Declaration of Conflicting Interests}

The Authors declare that there are no conflict of interest. 


\section{References}

Boccard SG, Pereira EA, Moir L, Van Hartevelt TJ, Kringelbach ML, FitzGerald JJ, Baker IW, Green $\mathrm{AL}$, Aziz TZ. 2014. Deep brain stimulation of the anterior cingulate cortex: targeting the affective component of chronic pain. Neuroreport. 25(2):83-88.

Bohr I, McDonald C, He J, Kerr S, Newton JL, Blamire AM. 2015. Brain oxygenation responses to an autonomic challenge: a quantitative $\mathrm{fMRI}$ investigation of the Valsalva manoeuvre. Age (Dordr). 37(5):91.

Bordoni B, Marelli F, Bordoni G. 2016. A review of analgesic and emotive breathing: a multidisciplinary approach. J Multidiscip Healthc. 9:97-102.

Borsook D, Veggeberg R, Erpelding N, Borra R, Linnman C, Burstein R, Becerra L. 2016. The Insula: A "Hub of Activity" in Migraine. Neuroscientist. 22(6):632-652.

Brugger M, Lutz K, Bronnimann B, Meier ML, Luechinger R, Barlow A, Jancke L, Ettlin DA. 2012. Tracing toothache intensity in the brain. J Dent Res. 91(2):156-160.

Chinthakanan S, Laosuwan K, Boonyawong P, Kumfu S, Chattipakorn N, Chattipakorn SC. 2018. Reduced heart rate variability and increased saliva cortisol in patients with TMD. Arch Oral Biol. 90:125-129.

Finkelmeyer A, He J, Maclachlan L, Blamire AM, Newton JL. 2018a. Intracranial compliance is associated with symptoms of orthostatic intolerance in chronic fatigue syndrome. PLoS One. 13(7):e0200068.

Finkelmeyer A, He J, Maclachlan L, Watson S, Gallagher P, Newton JL, Blamire AM. 2018b. Grey and white matter differences in Chronic Fatigue Syndrome - A voxel-based morphometry study. Neuroimage Clin. 17:24-30.

First M, Spitzer R, Gibbon M, Williams J. 2002. Structured Clinical Interview for DSM-IV-TR Axis I Disorders, Research Version, Patient Edition. (SCID-I/P). New York: Biometrics Research, New York State Psychiatric Institute.

Fisk JD, Ritvo PG, Ross L, Haase DA, Marrie TJ, Schlech WF. 1994. Measuring the functional impact of fatigue: initial validation of the fatigue impact scale. Clin Infect Dis. 18 Suppl 1:S79-83.

Friston KJ, Holmes AP, Worsley KJ, Poline J-P, Frith CD, Frackowiak RSJ. 1994. Statistical parametric maps in functional imaging: A general linear approach. Human Brain Mapping. 2(4):189-210.

Fukuda K, Straus SE, Hickie I, Sharpe MC, Dobbins JG, Komaroff A. 1994. The Chronic Fatigue Syndrome A Comprehensive Approach to Its Definition and Study. Ann Intern Med. 121:953959.

Gonzalez YM, Schiffman E, Gordon SM, Seago B, Truelove EL, Slade G, Ohrbach R. 2011. Development of a brief and effective temporomandibular disorder pain screening questionnaire. J Am Dent Assoc. 142(10):1183-1191. 
Greenspan JD, Slade GD, Bair E, Dubner R, Fillingim RB, Ohrbach R, Knott C, Diatchenko L, Liu Q, Maixner W. 2013. Pain sensitivity and autonomic factors associated with development of TMD: the OPPERA prospective cohort study. J Pain. 14(12 Suppl):T63-74 e61-66.

Harte SE, Ichesco E, Hampson JP, Peltier SJ, Schmidt-Wilcke T, Clauw DJ, Harris RE. 2016. Pharmacologic attenuation of cross-modal sensory augmentation within the chronic pain insula. Pain. 157(9):1933-1945.

Henderson LA, Macey PM, Macey KE, Frysinger RC, Woo MA, Harper RK, Alger JR, Yan-Go FL, Harper RM. 2002. Brain responses associated with the Valsalva maneuver revealed by functional magnetic resonance imaging. J Neurophysiol. 88:3477-3486.

Jason L, Evans M, Porter N, Brown M, Brown A, Hunnell J, Anderson V, Lerch A, De Meirleir K, Friedberg F. 2010. The development of a revised Canadian myalgic encephalomyelitis chronic fatigue syndrome case definition. Am J Biochem Biotechnol. 6:120-135.

Johnston S, Brenu EW, Staines D, Marshall-Gradisnik S. 2013. The prevalence of chronic fatigue syndrome/ myalgic encephalomyelitis: a meta-analysis. Clin Epidemiol. 5:105-110.

Khurana RK. 2014. Visceral sensitization in postural tachycardia syndrome. Clin Auton Res. 24(2):71-76.

Koteles F, Witthoft M. 2017. Somatosensory amplification - An old construct from a new perspective. J Psychosom Res. 101:1-9.

Light KC, Bragdon EE, Grewen KM, Brownley KA, Girdler SS, Maixner W. 2009. Adrenergic dysregulation and pain with and without acute beta-blockade in women with fibromyalgia and temporomandibular disorder. J Pain. 10(5):542-552.

Macey PM, Wu P, Kumar R, Ogren JA, Richardson HL, Woo MA, Harper RM. 2012. Differential responses of the insular cortex gyri to autonomic challenges. Auton Neurosci. 168(1-2):7281.

Maixner W, Greenspan JD, Dubner R, Bair E, Mulkey F, Miller V, Knott C, Slade GD, Ohrbach R, Diatchenko $L$ et al. 2011. Potential autonomic risk factors for chronic TMD: descriptive data and empirically identified domains from the OPPERA case-control study. J Pain. 12(11 Suppl):T75-91.

Maldjian JA, Laurienti PJ, Kraft RA, Burdette JH. 2003. An automated method for neuroanatomic and cytoarchitectonic atlas-based interrogation of fMRI data sets. Neuroimage. 19(3):12331239.

Martinez-Martinez LA, Mora T, Vargas A, Fuentes-Iniestra M, Martinez-Lavin M. 2014. Sympathetic nervous system dysfunction in fibromyalgia, chronic fatigue syndrome, irritable bowel syndrome, and interstitial cystitis: a review of case-control studies. J Clin Rheumatol. 20(3):146-150. 
Miller AH, Jones JF, Drake DF, Tian H, Unger ER, Pagnoni G. 2014. Decreased basal ganglia activation in subjects with chronic fatigue syndrome: association with symptoms of fatigue. PLoS One. 9(5):e98156.

Newton JL, Okonkwo O, Sutcliffe K, Seth A, Shin J, Jones DE. 2007. Symptoms of autonomic dysfunction in chronic fatigue syndrome. QJM. 100(8):519-526.

Pazo JH, Belforte JE. 2002. Basal Ganglia and Functions of the Autonomic Nervous System. Cell Mol Neurobiol. 22(5):645-654.

Puri BK, Jakeman PM, Agour M, Gunatilake KD, Fernando KA, Gurusinghe Al, Treasaden IH, Waldman AD, Gishen P. 2012. Regional grey and white matter volumetric changes in myalgic encephalomyelitis (chronic fatigue syndrome): a voxel-based morphometry $3 \mathrm{~T}$ MRI study. Br J Radiol. 85(1015):e270-273.

Robinson LJ, Durham J, MacLachlan LL, Newton JL. 2015. Autonomic function in chronic fatigue syndrome with and without painful temporomandibular disorder. Fatigue. 3(4):205-219.

Robinson LJ, Durham J, Newton JL. 2016. A systematic review of the comorbidity between Temporomandibular Disorders and Chronic Fatigue Syndrome. J Oral Rehabil. 43(4):306316.

Schmidt-Wilcke T, Kairys A, Ichesco E, Fernandez-Sanchez ML, Barjola P, Heitzeg M, Harris RE, Clauw DJ, Glass J, Williams DA. 2014. Changes in clinical pain in fibromyalgia patients correlate with changes in brain activation in the cingulate cortex in a response inhibition task. Pain Med. 15(8):1346-1358.

Seifert F, Schuberth N, De Col R, Peltz E, Nickel FT, Maihofner C. 2013. Brain activity during sympathetic response in anticipation and experience of pain. Hum Brain Mapp. 34(8):17681782.

Slade GD, Fillingim RB, Sanders AE, Bair E, Greenspan JD, Ohrbach R, Dubner R, Diatchenko L, Smith SB, Knott $C$ et al. 2013. Summary of findings from the OPPERA prospective cohort study of incidence of first-onset temporomandibular disorder: implications and future directions. J Pain. 14(12 Suppl):T116-124.

Sullivan M, Bishop S, Pivik J. 1995. The pain catastrophizing scale: development and validation. Psychol Assess. 7:524-532.

Timmers HJ, Wieling W, Soetekouw PM, Bleijenberg G, Van Der Meer JW, Lenders JW. 2002. Hemodynamic and neurohumoral responses to head-up tilt in patients with chronic fatigue syndrome. Clin Auton Res. 12(4):273-280.

Van Cauwenbergh D, Nijs J, Kos D, Van Weijnen L, Struyf F, Meeus M. 2014. Malfunctioning of the autonomic nervous system in patients with chronic fatigue syndrome: a systematic literature review. Eur J Clin Invest. 44(5):516-526. 
van der Schaaf ME, De Lange FP, Schmits IC, Geurts DEM, Roelofs K, van der Meer JWM, Toni I, Knoop H. 2017. Prefrontal Structure Varies as a Function of Pain Symptoms in Chronic Fatigue Syndrome. Biol Psychiatry. 81(4):358-365.

Younger JW, Shen YF, Goddard G, Mackey SC. 2010. Chronic myofascial temporomandibular pain is associated with neural abnormalities in the trigeminal and limbic systems. Pain. 149(2):222-228. 


\section{Figure Captions}

Figure 1. (A) The Valsalva-manoeuvre protocol used in the current study. During the manoeuvre, participants was provided with visual feedback to maintain an expiratory pressure of $40 \mathrm{mmHg}$ against a closed outlet. The protocol consisted of a 4-s preparation period, a 16-s Valsalva period and a 40 -s rest period, repeated 4 times. The rest period was divided into a 20 -sec post-Valsalva period for the purpose of analyses. (B). An example of an exhalation trace from a CFS+ patient during the entire functional run.

Figure 2. (A) Significant clusters from the F-test (all clusters: $p_{\mathrm{FWE}}<0.05$ ). Cross hair at the cluster in right putamen (MNI coordinates: $x=33, y=-13, z=-5$ ). (B) The axial slice (the same slice as in Panel A) illustrates the overlap (yellow) between anatomically defined left and right insular cortex (red) and the largest left and right clusters from the F-test (cyan).

Figure 3. Uncorrected clusters in the left hemisphere from the comparison between the CFS+ and CFS- groups. Cross hair at the cluster in left insular cortex (MNI coordinates: $x=-27, y=14, z=7$; $p=0.037$, significant after small volume correction). 
Table 1. Participant information for each cohort group.

\begin{tabular}{|c|c|c|c|c|c|c|c|}
\hline Group & $\begin{array}{c}\text { Females/ } \\
\text { Males }\end{array}$ & $\begin{array}{c}\text { Mean } \\
\text { age* } \\
\text { in years } \\
\text { [min max] }\end{array}$ & $\begin{array}{c}\text { Mean } \\
\text { Symptom } \\
\text { Duration* } \\
\text { in years } \\
\text { (SD) }\end{array}$ & $\begin{array}{l}\text { Median } \\
\text { TMD } \\
\text { screener } \\
\text { score } \\
\text { [min max] }\end{array}$ & $\begin{array}{c}\text { Mean } \\
\text { DSQ }^{* *} \\
(S D)\end{array}$ & $\begin{array}{l}\text { Mean } \\
\text { FIS** }^{*} \\
\text { (SD) }\end{array}$ & $\begin{array}{c}\text { Mean } \\
\text { PCQ }^{*} \\
\text { (SD) }\end{array}$ \\
\hline CFS+ & $15 / 1$ & $\begin{array}{l}43.9 \\
{[2654]}\end{array}$ & $\begin{array}{l}12.1 \\
(10.4)\end{array}$ & $\begin{array}{l}4.5 \\
{[37]}\end{array}$ & $\begin{array}{l}39.6 \\
(28.6)\end{array}$ & $\begin{array}{l}104.6 \\
(37.1)\end{array}$ & $\begin{array}{l}13.8 \\
(10.8)\end{array}$ \\
\hline CFS- & $17 / 9$ & $\begin{array}{l}46.7 \\
{[2368]}\end{array}$ & $\begin{array}{l}15.1 \\
(10.2)\end{array}$ & $\begin{array}{l}0.0 \\
{\left[\begin{array}{ll}0 & 2\end{array}\right]}\end{array}$ & $\begin{array}{l}18.8 \\
(14.1)\end{array}$ & $\begin{array}{l}83.6 \\
(29.8)\end{array}$ & $\begin{array}{l}10.2 \\
(9.9)\end{array}$ \\
\hline Control & $7 / 3$ & $\begin{array}{l}49.4 \\
{[2565]}\end{array}$ & $\mathrm{N} / \mathrm{A}$ & 0.0 & $2.7(3.8)$ & $\mathrm{N} / \mathrm{A}$ & $\mathrm{N} / \mathrm{A}$ \\
\hline
\end{tabular}

DSQ = pain-related items from the DePaul Symptom Questionnaire (Jason et al. 2010)( $\mathrm{n}=41$ ); FIS = Fatigue Impact Scale (Fisk et al. 1994) $(n=41) ; P C Q=$ Pain Catastrophizing Questionnaire (Sullivan et al. 1995) $(n=32)$; note that questionnaire data were not available for all patients

${ }^{*} \mathrm{CFS}+$ did not differ from CFS- in age, symptom duration or PCQ scores, all $p s>.34$

${ }^{* *}$ CFS+ differed from CFS- in DSQ scores; $t(39)=3.11, p=0.004$; and marginally for FIS scores; $t(39)=2.01, p=0.051$ 
Table 2. The brain structures and statistics for clusters from the whole-brain analyses. The cluster size is the number of contiguous voxels in that cluster. The cluster $p$ (unc) is the uncorrected $p$-value at the cluster-level. The peak $p$ (unc) is the uncorrected $p$-value of the voxel with the largest activation in that cluster, and the peak MNI coordinates refer to that voxel's position. For the $F$-test, all clusters were significant after whole-brain family-wise-error correction at the cluster-level ( $\left.p_{\mathrm{FWE}}<0.05\right)$. The values in parentheses refer to voxels that overlapped with anatomically defined insular cortex. For the different group comparisons, clusters with uncorrected $p<0.05$ at the cluster-level are also reported for exploratory purposes. Two of the clusters were significant after small volume correction (SVC, reported in parentheses).

\begin{tabular}{|c|c|c|c|c|c|c|c|}
\hline \multirow[b]{2}{*}{ Structure } & \multirow[b]{2}{*}{ Hemisphere } & \multirow[b]{2}{*}{$\begin{array}{l}\text { cluster size } \\
\text { (voxels) }\end{array}$} & \multirow[b]{2}{*}{$\begin{array}{l}\text { cluster } p \\
\quad \text { (unc) }\end{array}$} & \multirow[b]{2}{*}{$\begin{array}{l}\text { peak } p \\
\text { (unc) }\end{array}$} & \multicolumn{3}{|c|}{ Peak MNI coordinates } \\
\hline & & & & & $x$ & $y$ & $z$ \\
\hline \multicolumn{8}{|l|}{$F$-test } \\
\hline $\begin{array}{l}\text { Putamen } \\
\text { (Insula) }\end{array}$ & Right & $\begin{array}{l}305 \\
(23)\end{array}$ & $<.001$ & $<.001$ & 33 & -13 & -5 \\
\hline $\begin{array}{l}\text { Putamen } \\
\text { (Insula) }\end{array}$ & Left & $\begin{array}{l}369 \\
(59)\end{array}$ & $<.001$ & $<.001$ & -30 & -4 & -2 \\
\hline Thalamus & Bilateral & 773 & $<.001$ & $<.001$ & 3 & -25 & 4 \\
\hline $\begin{array}{l}\text { Superior } \\
\text { Frontal Gyrus }\end{array}$ & Right & 209 & $<.001$ & $<.001$ & 9 & 53 & 1 \\
\hline $\begin{array}{l}\text { Superior } \\
\text { Frontal Gyrus }\end{array}$ & Left & 237 & $<.001$ & $<.001$ & -9 & 47 & -8 \\
\hline $\begin{array}{l}\text { Inferior Frontal } \\
\text { Gyrus }\end{array}$ & Left & 115 & $<.001$ & $<.001$ & -51 & 14 & 4 \\
\hline \multicolumn{8}{|l|}{ CFS+ vs CFS- } \\
\hline Insular Cortex & Left & 26 & .108 & $\begin{array}{c}<.001 \\
(.037 \mathrm{svc})\end{array}$ & -27 & 14 & 7 \\
\hline \multicolumn{8}{|l|}{ CFS+ vs Control } \\
\hline Caudate & Left & 49 & .034 & $\begin{array}{c}<.001 \\
(.016 \mathrm{svc})\end{array}$ & -12 & 5 & 1 \\
\hline \multicolumn{8}{|l|}{ CFS- vs Control } \\
\hline $\begin{array}{l}\text { Cingulate } \\
\text { Gyrus }\end{array}$ & Right & 63 & .018 & $<.001$ & 18 & -31 & 34 \\
\hline Parietal Lobe & Right & 63 & .018 & $<.001$ & 27 & -76 & 37 \\
\hline Cuneus & Left & 56 & .024 & $<.001$ & -15 & -88 & 4 \\
\hline Temporal Lobe & Right & 77 & .010 & $<.001$ & 33 & -37 & 13 \\
\hline $\begin{array}{l}\text { Cingulate } \\
\text { Gyrus }\end{array}$ & Right & 44 & .042 & $<001$ & 9 & -46 & 25 \\
\hline
\end{tabular}




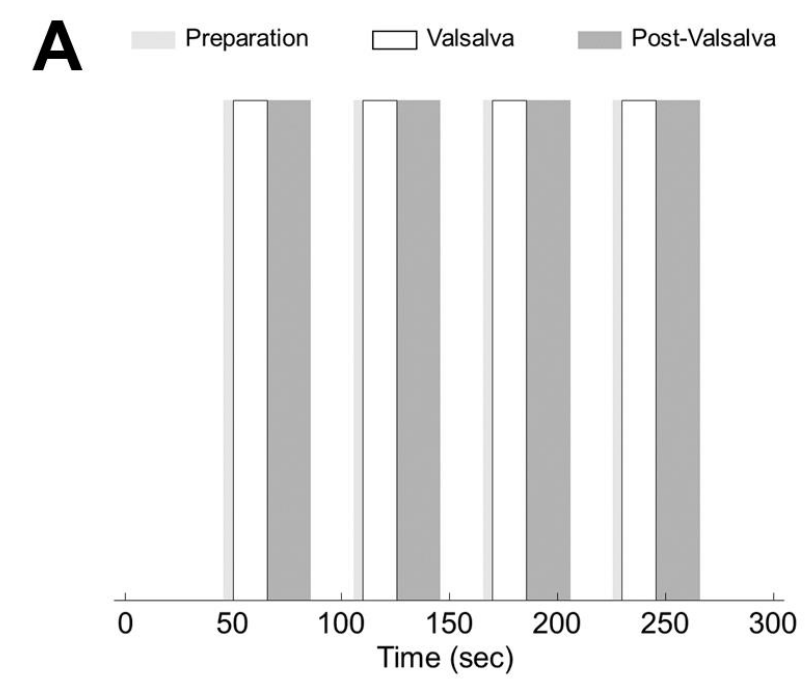

B

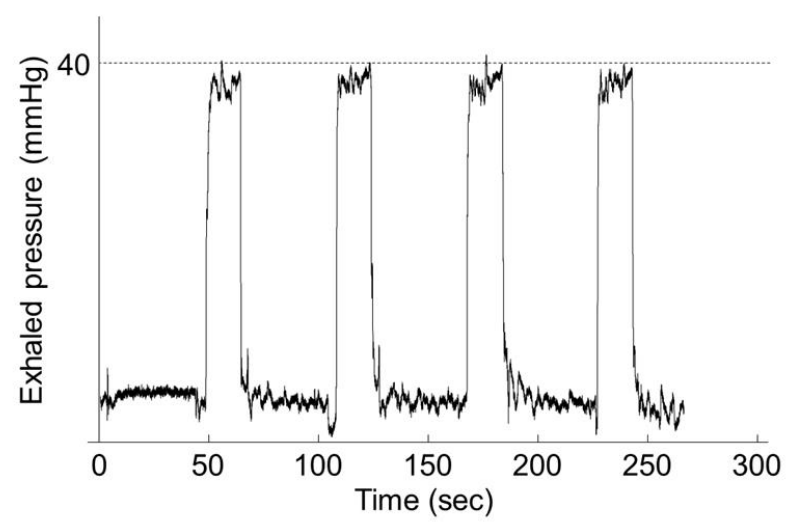

Figure 1. (A) The Valsalva-manoeuvre protocol used in the current study. During the manoeuvre, participants was provided with visual feedback to maintain an expiratory pressure of $40 \mathrm{mmHg}$ against a closed outlet. The protocol consisted of a 4-s preparation period, a 16-s Valsalva period and a 40 -s rest period, repeated 4 times. The rest period was divided into a 20 -sec post-Valsalva period for the purpose of analyses. (B). An example of an exhalation trace from a CFS+ patient during the entire functional run. 


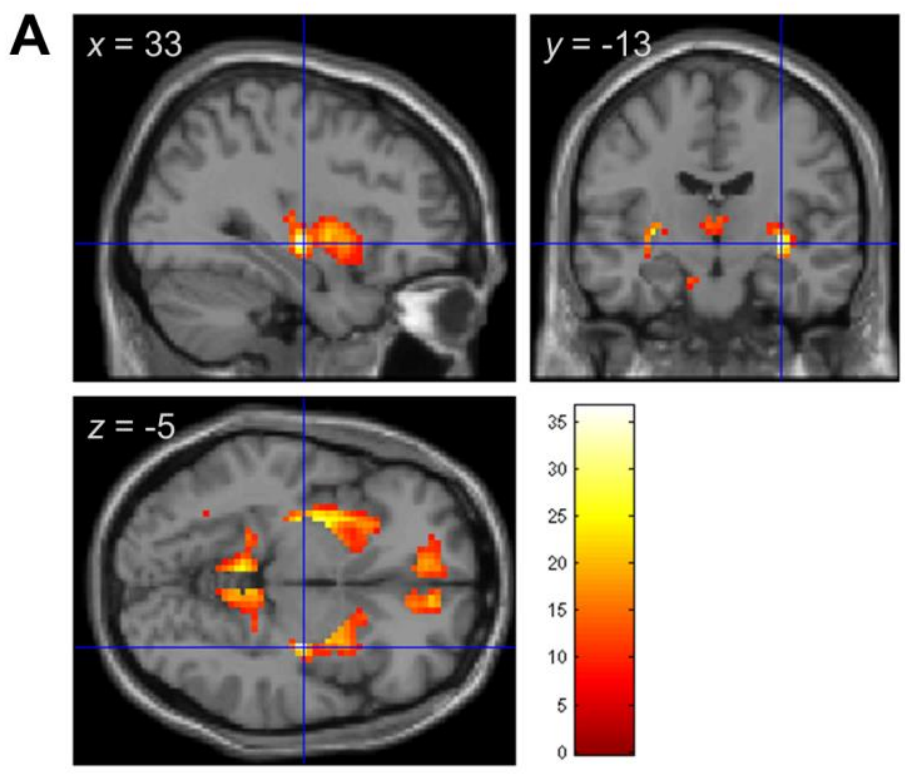

B

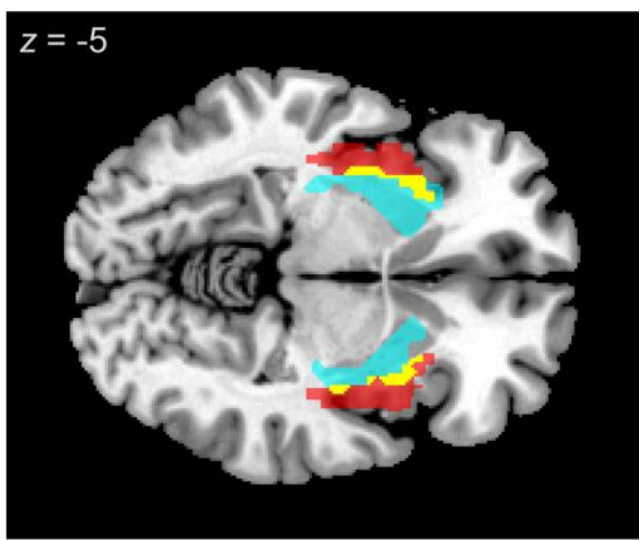

Figure 2. (A) Significant clusters from the F-test (all clusters: $p_{\mathrm{FWE}}<0.05$ ). Cross hair at the cluster in right putamen (MNI coordinates: $x=33, y=-13, z=-5$ ). (B) The axial slice (the same slice as in Panel A) illustrates the overlap (yellow) between anatomically defined left and right insular cortex (red) and the largest left and right clusters from the F-test (cyan). 

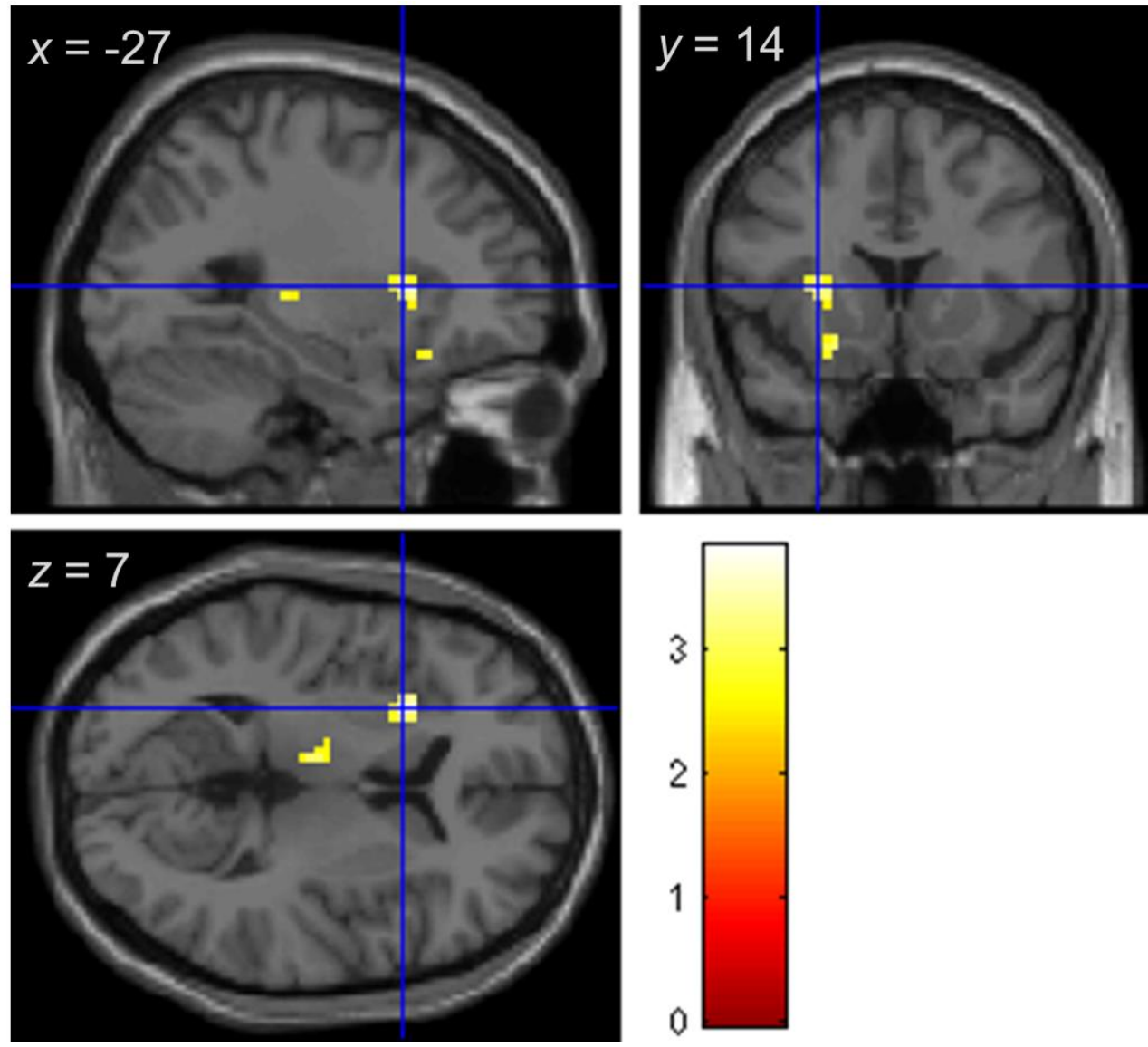

Figure 3. Uncorrected clusters in the left hemisphere from the comparison between the CFS+ and CFS-groups. Cross hair at the cluster in left insular cortex (MNI coordinates: $x=-27, y=14, z=7$; $p=0.037$, significant after small volume correction). 